\title{
Contraction Mapping Theory and Approach to LMI-Based Stability Criteria of T-S Fuzzy Impulsive Time-Delays Integrodifferential Equations
}

\author{
Ruofeng Rao' ${ }^{1}$ and Shouming Zhong ${ }^{2}$ \\ ${ }^{1}$ Department of Mathematics, Chengdu Normal University, Chengdu 61130, China \\ ${ }^{2}$ Research Institute of Mathematics, Chengdu Normal University, Chengdu 61130, China \\ Correspondence should be addressed to Ruofeng Rao; ruofengrao@163.com
}

Received 1 September 2016; Accepted 17 November 2016

Academic Editor: Enrique Llorens-Fuster

Copyright (C) 2016 R. Rao and S. Zhong. This is an open access article distributed under the Creative Commons Attribution License, which permits unrestricted use, distribution, and reproduction in any medium, provided the original work is properly cited.

In this paper, Banach fixed point theorem is employed to derive LMI-based exponential stability of impulsive Takagi-Sugeno (TS) fuzzy integrodifferential equations, originated from Cohen-Grossberg Neural Networks (CGNNs). As far as we know, Banach fixed point theorem is rarely employed to derive LMI criteria for T-S fuzzy CGNNs, and this inspires our present work. It is worth mentioning that the conditions on the behavior functions are weaker than those of existing results, and the formulated contraction mapping and fixed point technique are different from those of previous literature. Even a corollary of our main result improves one of existing main results due to extending linear function to nonlinear function. Besides, the LMI-based criteria are programmable for computer MATLAB LMI toolbox. Moreover, an analytical table and a numerical example are presented to illustrate the advantage, feasibility, and effectiveness of the proposed methods.

\section{Introduction}

In this paper, we consider a class of integrodifferential equations, which is originated from Cohen-Grossberg Neural Networks (CGNNs). CGNNs model was proposed originally by Cohen-Grossberg in 1983 [1]. Since then, the stability analysis of CGNNs has attracted extensive attentions owing to its wide applications. Lyapunov function method is a common technique which derived stability criteria of various time-delays dynamic systems [2-13]. However, any method has its limitations. Fixed point technique may be one of common replacements. Brouwer fixed point theorem and Schauder fixed point theorem were always applied to stability analysis of various CGNNs models [14-17]. Of course, Banach fixed point theorem was also employed to derive the stability criteria of various CGNNs models [17-22]. But for most of existing literature, either using the same inverse function translates CGNNs model into another model similar as follows (see, e.g., [17, 19-21]),

$$
\begin{aligned}
& x_{i}^{\prime}(t)=-b_{i}\left(u^{-1}\left(x_{i}(t)\right)\right)+\sum_{i=1}^{n}\left[b_{i j}(t) f_{j}\left(u^{-1}\left(x_{j}(t)\right)\right)\right. \\
& \left.\quad+c_{i j} f_{j}\left(u^{-1}\left(x_{j}(t-\tau(t))\right)\right)\right]
\end{aligned}
$$

or the stability criteria are too complex [22, Theorem 3.1], which cannot be programmable for computer software while practical engineering is often involved in large-scale computing. Of course, both methods and results of [17, 19-22] are good and referential. But, in order to innovate, we have to find 
another way. In [18], a LMI-based stability criterion was given for the following CGNNs:

$$
\begin{aligned}
& \frac{d x_{j}(t)}{d t}=-a_{j}\left(x_{j}(t)\right)\left\{b_{j}\left(x_{j}(t)\right)\right. \\
& \left.-\sum_{k=1}^{n}\left[c_{j k} f_{k}\left(x_{k}(t)\right)+d_{j k} g_{k}\left(x_{k}\left(t-\tau_{j}(t)\right)\right)\right]\right\}, \\
& t \geqslant 0, t \neq t_{i}, \\
& \Delta x_{j}\left(t_{i}\right)=x_{j}\left(t_{i}^{+}\right)-x_{j}\left(t_{i}\right)=\rho_{j i}\left(x_{j}\left(t_{i}\right)\right), \\
& x_{j}(\theta)=\phi_{j}(\theta), \quad-\tau \leqslant \theta \leqslant 0 .
\end{aligned}
$$

Theorem A (see [18, Theorem 4]). If (H1)-(H4) are satisfied and there exists a positive constant $\alpha<1$ such that the following LMI condition holds,

$$
C \bar{F} \widetilde{A}+D \bar{G} \widetilde{A}+H+\mu \Gamma+C F \bar{A}+D G \bar{A} \leqslant \alpha \Gamma \bar{A},
$$

then System (2) is globally exponentially stable, where the four conditions are proposed as follows.

(H1) For any $j \in \mathcal{N}$, there exist constants $\bar{F}_{j}>0, \bar{G}_{j}>0$, $F_{j}>0$, and $G_{j}>0$ such that

$$
\begin{gathered}
\left|f_{j}(r)\right| \leqslant \bar{F}_{j}, \\
\left|g_{j}(r)\right| \leqslant \bar{G}_{j}, \\
\left|f_{j}(r)-f_{j}(s)\right| \leqslant F_{j}|r-s|, \\
\left|g_{j}(r)-g_{j}(s)\right| \leqslant G_{j}|r-s|,
\end{gathered}
$$

$$
\forall r, s \in R \text {. }
$$

(H2) For any $j \in \mathcal{N}, a_{j}(\cdot)$ is differentiable, and there exists $a$ constant $\tilde{a}_{j}$ such that

$$
\begin{aligned}
0 & <a_{j}(r) \leqslant \bar{a}_{j}, \\
\left|a_{j}^{\prime}(r)\right| & \leqslant \widetilde{a}_{j},
\end{aligned}
$$

$$
r \in R \text {. }
$$

(H3) There exist nonnegative constants $\tilde{h}_{j i}$ such that

$$
\begin{aligned}
&\left|\rho_{j i}(r)-\rho_{j i}(s)\right| \leqslant \tilde{h}_{j i}|r-s|, \\
& \forall r, s \in R, j \in \mathcal{N}, i=1,2, \ldots
\end{aligned}
$$

(H4) For $j \in \mathcal{N}$, there exist $\Upsilon_{j}(t)$ and a constant $\gamma_{j}>0$ such that

$$
\begin{gathered}
\Upsilon_{j}(t) \geqslant \gamma_{j}, \quad \forall t \geqslant 0, \\
a_{j}(r) b_{j}(r)=\Upsilon_{j}(t) r, \quad r \in R .
\end{gathered}
$$

Remark 1. Denoting $\chi_{i}(s)=a_{i}(s) b_{i}(s)$, we know from [18, (H4)] that $\chi_{i}(s)$ can only be a linear function on $s$. So it is the main objective of this paper to make up the deficiency. Below, we try to make the condition of $\chi_{i}(s)$ better so that $\chi_{i}(s)$ may be a nonlinear function on $s$.

All the good results and methods in existing literature, particularly in [17, 19-22], inspired our current work. Of course, we cannot imitate (1) again, and the condition [18, (H4)] should be replaced by a new suitable condition. Below, we shall propose a new condition which is better than $[18,(\mathrm{H} 4)]$. Due to the changes of conditions, we have to formulate new contraction mapping to derive new LMIbased exponential stability criterion for Takagi-Sugeno (T-S) fuzzy impulsive CGNNs with discrete and distributed delays. This is one of main innovations of this paper. In addition, we shall admit weaker condition on the behavior functions than that of existing literature (e.g., [17, 19-22]) published from 2007 to 2016. Moreover, a corollary of our main result is better than [18, Theorem 3.1] due to the above reasons.

Remark 2. Due to the weaker condition on the behavior functions, both our results and methods are novelty (see below "Remark 13" and "Table 1" for details).

For convenience's sake, we introduce the following standard notations.

(i) $L=\left(l_{i j}\right)_{n \times n}>0(<0)$ is a positive (negative) definite matrix; that is, $y^{T} L y>0(<0)$ for any $0 \neq y \in R^{n}$.

(ii) $L=\left(l_{i j}\right)_{n \times n} \geqslant 0(\leqslant 0)$ is a semipositive (seminegative) definite matrix; that is, $y^{T} L y \geqslant 0(\leqslant 0)$ for any $y \in R^{n}$.

(iii) $L \in\left[L_{*}, L^{*}\right]$ implies that $l_{i j *} \leqslant l_{i j} \leqslant l_{i j}^{*}$ for all $i, j$ with $L=\left(l_{i j}\right)_{n \times n}, L_{*}=\left(l_{i j *}\right)_{n \times n}$, and $L^{*}=\left(l_{i j}^{*}\right)_{n \times n}$.

(iv) $L_{1} \geqslant L_{2}\left(L_{1} \leqslant L_{2}\right)$ means matrix $\left(L_{1}-L_{2}\right)$ is a semipositive (seminegative) definite matrix.

(v) $L_{1}>L_{2}\left(L_{1}<L_{2}\right)$ means matrix $\left(L_{1}-L_{2}\right)$ is a positive (negative) definite matrix.

(vi) $\lambda_{\max }(\Phi), \lambda_{\min }(\Phi)$ denotes the largest and smallest eigenvalue of matrix $\Phi$, respectively.

(vii) Denote $|L|=\left(\left|l_{i j}\right|\right)_{n \times n}$ for any matrix $L=\left(l_{i j}\right)_{n \times n}$.

(viii) $|u|=\left(\left|u_{1}\right|,\left|u_{2}\right|, \ldots,\left|u_{n}\right|\right)^{T}$ for any vector $u=$ $\left(u_{1}, u_{2}, \ldots, u_{n}\right)^{T} \in R^{n}$.

(ix) $u \leqslant(\geqslant) v$ implies that $u_{i} \leqslant(\geqslant) v_{i}, \forall i$, and $u<(>)$ $v$ implies that $u_{i}<(>) v_{i}, \forall i$, for any vectors $u=$ $\left(u_{1}, u_{2}, \ldots, u_{n}\right)^{T} \in R^{n}$ and $v=\left(v_{1}, v_{2}, \ldots, v_{n}\right)^{T} \in R^{n}$.

(x) $I$ is identity matrix with compatible dimension.

\section{Preliminaries}

Cohen-Grossberg Neural Networks (CGNNs) with discrete and distributed delays have been investigated in many papers 
TABLE 1: Comparing our Theorem 8 and Corollary 10 with other existing criteria on CGNNs derived by fixed point theorems.

\begin{tabular}{lcccc}
\hline & Fixed point theorems & Continuity of $b_{i}$ & Differentiability of $b_{i}$ & Programmabilit $y$ of criteria \\
\hline Our Theorem 8 (Corollary 10) & Contraction mapping theorem & Unnecessary & Unnecessary & Yes, LMI-based \\
[22, Theorem 3.1] & Contraction mapping theorem & Yes, necessary & {$[22,($ A2) $]$} & No \\
[19, Theorem 1] & Contraction mapping theorem & Yes, necessary & {$[19,($ A2) $]$} & No \\
[20, Theorems 1 and 2] & Contraction mapping theorem & Yes, necessary & Yes, necessary & No \\
[17, Theorems 1-3] & Brouwer fixed point theorem & Yes, necessary & {$[17,($ H3) $)$} & No \\
[21, Theorem 3.1] & Contraction mapping theorem & Yes, necessary & Yes, necessary & No \\
\hline
\end{tabular}

Conditions $[19,(\mathrm{~A} 2)],[22,(\mathrm{~A} 2)]$, and $[17,(\mathrm{H} 3)]$ are similar to $\left(b_{i}(s)-b_{i}(t)\right) /(s-t) \geqslant$ constant

[23-28]. Consider the following impulsive CGNNs with discrete and distributed delays,

$$
\begin{aligned}
& \frac{d x(t)}{d t}=-A(x(t))[B(x(t))-C f(x(t)) \\
& \left.-D g(x(t-\tau(t)))-E \int_{t-\delta(t)}^{t} \eta(x(s)) d s\right], \\
& \quad t \geqslant 0, t \neq t_{k}, \\
& x\left(t_{k}^{+}\right)-x\left(t_{k}^{-}\right)=\rho\left(x\left(t_{k}\right)\right), \quad k=1,2, \ldots
\end{aligned}
$$

with the initial condition

$$
x(s)=\phi(s), \quad s \in[-\tau, 0],
$$

where $x=\left(x_{1}, x_{2}, \ldots, x_{n}\right) \in R^{n}$ with $x_{i}(t)$ being the state variables of the $i$ th neuron at time $t$. The neuron active functions $f(x)=\left(f_{1}\left(x_{1}(t)\right), f_{2}\left(x_{2}(t)\right), \ldots, f_{n}\left(x_{n}(t)\right)\right)^{T} \in$ $R^{n}$ for any given $t \in R$. $A(x(t))=\operatorname{diag}\left(a_{1}\left(x_{1}(t)\right)\right.$, $\left.a_{2}\left(x_{2}(t)\right), \ldots, a_{n}\left(x_{n}(t)\right)\right)$ is $n$-dimension diagonal matrix with $a_{i}\left(x_{i}(t)\right)$ representing an amplification function, and $B(x(t))=\left(b_{1}\left(x_{1}(t)\right), \ldots, b_{n}\left(x_{n}(t)\right)\right)^{T} \in R^{n}$ with $b_{j}\left(x_{j}(t)\right)$ being an appropriately behavior function. $C=\left(c_{i j}\right)_{n \times n}$, $D=\left(d_{i j}\right)_{n \times n}$, and $E=\left(e_{i j}\right)_{n \times n}$ are the connection weight matrices with $c_{i j}, d_{i j}, e_{i j}$ representing the strengths of connectivity between cells $i$ and $j$ at time $t$. Vector functions $f(x(t))=\left(f_{1}\left(x_{1}(t)\right), \ldots, f_{n}\left(x_{n}(t)\right)\right)^{T}, g(x(t))=$ $\left(g_{1}\left(x_{1}(t-\tau(t))\right), \ldots, g_{n}\left(x_{n}(t-\tau(t))\right)\right)^{T}$, and $\eta(x(s))=$ $\left(\eta_{1}\left(x_{1}(s)\right), \ldots, \eta_{n}\left(x_{n}(s)\right)\right)^{T}$ with activation functions $f_{j}(\cdot)$, $g_{j}(\cdot), \eta_{j}(\cdot)$ telling how the $j$ th neuron reacts to the input. $\tau(t)$ is discrete delay, and $\delta(t)$ is distributed time-delay. $\tau$ is a positive constant with $\delta(t), \tau(t) \in[0, \tau] \cdot \rho(x(t))=$ $\left(\rho_{1}\left(x_{1}(t)\right), \ldots, \rho_{n}\left(x_{n}(t)\right)\right)^{T}$ denotes the impulsive function, and the fixed impulsive moments $t_{k}(k=1,2, \ldots)$ satisfy $0<t_{1}<t_{2}<\cdots$ with $\lim _{k \rightarrow+\infty} t_{k}=+\infty . x\left(t_{k}^{+}\right)$and $x\left(t_{k}^{-}\right)$ stand for the right-hand and left-hand limit of $x(t)$ at time $t_{k}$, respectively. Further, suppose that

$$
\begin{aligned}
& x\left(t_{k}^{-}\right)=\lim _{t \rightarrow t_{k}^{-}} x(t)=x\left(t_{k}\right), \\
& y\left(t_{k}^{-}\right)=\lim _{t \rightarrow t_{k}^{-}} y(t)=y\left(t_{k}\right),
\end{aligned}
$$

$$
k=1,2, \ldots
$$

In addition, we assume that $\phi(s) \in \mathscr{C}([-\tau, 0] ; R)$, where $\mathscr{C}([-\tau, 0] ; R)$ is the space of all the continuous functions defined on $[-\tau, 0]$.

Since practice has shown that fuzzy logic theory is an efficient approach to deal with the analysis and synthesis problems for complex nonlinear system, the fuzzy model is far more important than stochastic model [29-31]. Among various kinds of fuzzy methods, Takagi-Sugeno (T-S) fuzzy models provide a successful method to describe certain complex nonlinear systems using some local linear subsystems.

Below, we describe the T-S fuzzy mathematical model with time-delay as follows.

Fuzzy Rule j. IF $\omega_{1}(t)$ is $\mu_{j 1}, \ldots, \omega_{s^{*}}(t)$ is $\mu_{j s^{*}}$ THEN

$$
\begin{aligned}
& \frac{d x(t)}{d t}=-A(x(t))\left[B(x(t))-C_{j} f(x(t))\right. \\
& \left.-D_{j} g(x(t-\tau(t)))-E_{j} \int_{t-\delta(t)}^{t} \eta(x(s)) d s\right], \\
& \quad t \geqslant 0, t \neq t_{k}, \\
& x\left(t_{k}^{+}\right)-x\left(t_{k}^{-}\right)=\rho\left(x\left(t_{k}\right)\right), \quad k=1,2, \ldots,
\end{aligned}
$$

where $\omega_{k}(t)\left(k=1,2, \ldots, s^{*}\right)$ is the premise variable, $\mu_{j k}\left(j=1,2, \ldots, r ; k=1,2, \ldots, s^{*}\right)$ is the fuzzy set that is characterized by membership function, $r$ is the number of the IF-THEN rules, and $s^{*}$ is the number of the premise variables. By way of a standard fuzzy inference method, System (11) is inferred as follows:

$$
\begin{aligned}
& \frac{d x(t)}{d t}=-A(x(t))\left[B(x(t))-\sum_{j=1}^{r^{*}} h_{j}(\omega(t))\right. \\
& \cdot\left(C_{j} f(x(t))+D_{j} g(x(t-\tau(t)))\right. \\
& \left.\left.+E_{j} \int_{t-\delta(t)}^{t} \eta(x(s)) d s\right)\right], \quad t \geqslant 0, t \neq t_{k}, \\
& x\left(t_{k}^{+}\right)-x\left(t_{k}^{-}\right)=\rho\left(x\left(t_{k}\right)\right), \quad k=1,2, \ldots,
\end{aligned}
$$

where $\omega(t)=\left[\omega_{1}(t), \omega_{2}(t), \ldots, \omega_{s}(t)\right], h_{j}(\omega(t))=w_{j}(\omega(t)) /$ $\sum_{k=1}^{r} w_{k}(\omega(t))$, and $w_{j}(\omega(t)): R^{s^{*}} \rightarrow[0,1]\left(j=1,2, \ldots, r^{*}\right)$ is the membership function of the system with respect to 
the fuzzy rule $j . h_{j}$ can be regarded as the normalized weight of each IF-THEN rule, satisfying $h_{j}(\omega(t)) \geqslant 0$ and $\sum_{j=1}^{r^{*}} h_{j}(\omega(t))=1$.

Throughout this paper, we assume that $B(0)=f(0)=$ $g(0)=\eta(0)=0 \in R^{n}$ and the following.

(A1) $f_{i}, g_{i}, \eta_{i}, \rho_{i}$ all are Lipschitz continuous functions with Lipschitz constants $F_{i}, G_{i}, Q_{i}, H_{i}$, respectively. In addition, there are positive constants $F^{*}, G^{*}, Q^{*}$ such that

$$
\begin{aligned}
& \left|f_{i}(s)\right| \leqslant F^{*}, \\
& \left|g_{i}(s)\right| \leqslant G^{*}, \\
& \left|\eta_{i}(s)\right| \leqslant Q^{*} .
\end{aligned}
$$

(A2) There is positive definite diagonal matrix $\bar{A}$ such that $A(x(t)) \in[0, \bar{A}]$, and $a_{i}(s)$ is Lipschitz continuous with Lipschitz constant $\tilde{a}_{i}$, for all $s \in \mathbb{R}, i=$ $1,2, \ldots, n$, where $A(x(t))=\operatorname{diag}\left(a_{1}\left(x_{1}(t)\right), a_{2}\left(x_{2}(t)\right)\right.$, $\left.\ldots, a_{n}\left(x_{n}(t)\right)\right)$ is $n$-dimension diagonal matrix.

(A3) For each $i$, there exists a constant $m_{i}>0$ such that the function $\left(a_{i}(s) b_{i}(s)-m_{i} s\right)$ is Lipschitz continuous with the Lipschitz constant $l_{i}$.

Remark 3. There are a large number of functions $a_{i}(\cdot), b_{i}(\cdot)$ satisfying (A2) and (A3).

For example, we denote

$$
\varphi(s)= \begin{cases}\frac{\arctan s}{s}, & s \neq 0, \\ 1, & s=0 .\end{cases}
$$

Obviously, $\varphi(s)$ is continuous for all $s \in R$ so that $\max _{s \in R} \varphi(s)$ exists, and $\max _{s \in R} \varphi(s) \geqslant \varphi(0)>0$. Let $m_{i}=1+\max _{s \in R} \varphi(s)$, and then the constant $m_{i}>1$. Next, let

$$
\begin{aligned}
& a_{i}(s)=m_{i}+\varphi(s), \\
& b_{i}(s)=s,
\end{aligned}
$$

and then

$$
\begin{aligned}
& a_{i}(s) b_{i}(s)=m_{i} s+s \varphi(s), \\
& \quad 0<1 \leqslant a_{i}(s) \leqslant 2 m_{i}, b_{i}(0)=0 .
\end{aligned}
$$

Hence,

$$
a_{i}(s) b_{i}(s)-m_{i} s= \begin{cases}\arctan s, & s \neq 0 \\ 0, & s=0\end{cases}
$$

and it is easy to prove that the above function $\left(a_{i}(s) b_{i}(s)-m_{i} s\right)$ is differentiable, and $\left(a_{i}(s) b_{i}(s)-m_{i} s\right)^{\prime} \leqslant 1$ for all $s \in R$. So we can set $l_{i}=1$ such that

$$
\begin{aligned}
& \left|\left(a_{i}(s) b_{i}(s)-m_{i} s\right)-\left(a_{i}(t) b_{i}(t)-m_{i} t\right)\right| \\
& \quad \leqslant\left[\sup _{s \in R}\left(a_{i}(s) b_{i}(s)-m_{i} s\right)^{\prime}\right]|s-t| \leqslant l_{i}|s-t| .
\end{aligned}
$$

In addition, we can prove that $a_{i}(s)=m_{i}+\varphi(s)$ is also Lipschitz continuous. Indeed,

$$
\left|a_{i}(s)-a_{i}(t)\right|=|\varphi(s)-\varphi(t)| .
$$

Below, we only need to prove $\varphi(s)$ is Lipschitz continuous. Firstly, we claim that $\varphi(s)$ is differentiable for all $s \in R$. In fact, we can get by L'Hospital's rule and $s=\tan r$

$$
\begin{aligned}
\varphi^{\prime}(0) & =\lim _{s \rightarrow 0} \frac{\varphi(s)-1}{s-0}=\lim _{s \rightarrow 0} \frac{\arctan s / s-1}{s} \\
& =\lim _{s \rightarrow 0}\left(\frac{\arctan s}{s}\right)^{\prime}=\lim _{s \rightarrow 0} \frac{s /\left(1+s^{2}\right)-\arctan s}{s^{2}} \\
& =\lim _{r \rightarrow 0} \frac{\tan r /\left(1+\tan ^{2} r\right)-r}{\tan ^{2} r} \\
& =\lim _{r \rightarrow 0} \frac{(1 / 2) \sin (2 r)-r}{r^{2}}=\lim _{r \rightarrow 0} \frac{\cos (2 r)-1}{2 r} \\
& =\lim _{r \rightarrow 0} \frac{-2 \sin (2 r)}{2}=0 .
\end{aligned}
$$

Hence, $\varphi(s)$ is differentiable for all $s \in R$, and $\sup _{s \in R}\left|\varphi^{\prime}(s)\right|=\sup _{s \neq 0}\left|(\arctan s / s)^{\prime}\right|$. Further, Lagrangian differential mean value theorem derives

$$
|\varphi(s)-\varphi(t)| \leqslant|s-t| \sup _{s \neq 0}\left|\left(\frac{\arctan s}{s}\right)^{\prime}\right| .
$$

Owing to

$$
\begin{aligned}
& \lim _{s \rightarrow 0}\left(\frac{\arctan s}{s}\right)^{\prime}=0, \\
& \lim _{s \rightarrow \infty}\left(\frac{\arctan s}{s}\right)^{\prime}=\lim _{s \rightarrow \infty} \frac{s /\left(1+s^{2}\right)-\arctan s}{s^{2}}=0,
\end{aligned}
$$

we can conclude that the continuous function $(\arctan s / s)^{\prime}$ is bounded in all $s \neq 0$, which implies that $\sup _{s \neq 0}\left|(\arctan s / s)^{\prime}\right|$ is a positive constant, and hence both $\varphi(s)$ and $a_{i}(s)$ are Lipschitz continuous for all $s \in R$. This has proved that $a_{i}(s)$ and $b_{i}(s)$ defined as (15) satisfy (A2) and (A3).

Remark 4. Let $\chi_{i}(s)=a_{i}(s) b_{i}(s)$; we know that condition (A3) admits that $\chi_{i}(s)$ is a nonlinear function on $s$ while $[18,(\mathrm{H} 4)]$ derives that $\chi_{i}(s)$ can only be a linear function on $s$.

Remark 5. Since (H4) is replaced with (A3), the methods of [18] cannot be applied to this paper, and we have to formulate new contraction mapping, different from [18].

Definition 6. Impulsive fuzzy CGNNs (12) with initial condition (9) are said to be globally exponentially stable if, for any initial condition $\phi(s) \in \mathscr{C}\left([-\tau, 0], R^{n}\right)$, there exists a pair of positive constants $a$ and $b$ such that

$$
\|x(t ; s, \phi)\| \leqslant b e^{-a t}, \quad \forall t>0,
$$

where the norm $\|x(t)\|=\left(\sum_{i=1}^{n}\left|x_{i}(t)\right|^{2}\right)^{1 / 2}$.

Lemma 7 (contraction mapping theorem, see [32]). Letting $P$ be a contraction operator on a complete metric space $\Theta$, then there exists a unique point $\theta \in \Theta$ for which $P(\theta)=\theta$. 


\section{Main Results}

If (A1)-(A3) hold, we can derive the following main result.

Theorem 8. Impulsive fuzzy CGNNs (12) with initial condition (9) are global exponential stability if there exists a positive number $\alpha<1$, satisfying the following LMI condition:

$$
\begin{aligned}
L+\bar{A} & \sum_{j=1}^{r^{*}}\left|C_{j}\right| F+F^{*} \mathscr{A} \sum_{j=1}^{r^{*}}\left|C_{j}\right|+\bar{A} \sum_{j=1}^{r^{*}}\left|D_{j}\right| G \\
& +G^{*} \mathscr{A} \sum_{j=1}^{r^{*}}\left|D_{j}\right| \\
& +\tau\left(\bar{A} \sum_{j=1}^{r^{*}}\left|E_{j}\right| Q+Q^{*} \mathscr{A} \sum_{j=1}^{r^{*}}\left|E_{j}\right|\right)+\frac{1}{\mu} H+M H \\
& -\alpha M<0,
\end{aligned}
$$

where $\mu=\inf _{k=1,2, \ldots}\left(t_{k+1}-t_{k}\right)>0$, and

$$
\begin{aligned}
& F=\operatorname{diag}\left(F_{1}, F_{2}, \ldots, F_{n}\right), \\
& G=\operatorname{diag}\left(G_{1}, G_{2}, \ldots, G_{n}\right),
\end{aligned}
$$

$$
\begin{aligned}
& Q=\operatorname{diag}\left(\mathrm{Q}_{1}, \mathrm{Q}_{2}, \ldots, \mathrm{Q}_{n}\right), \\
& H=\operatorname{diag}\left(H_{1}, H_{2}, \ldots, H_{n}\right), \\
& \mathscr{A}=\operatorname{diag}\left(\widetilde{a}_{1}, \widetilde{a}_{2}, \ldots, \widetilde{a}_{n}\right), \\
& M=\operatorname{diag}\left(m_{1}, \ldots, m_{n}\right), \\
& L=\operatorname{diag}\left(l_{1}, \ldots, l_{n}\right) .
\end{aligned}
$$

Proof. First of all, we need to formulate integral equations equivalent to (12).

Denote, for convenience, $\zeta_{i}(s) \triangleq a_{i}(s) b_{i}(s)-m_{i} s$, and then $\zeta(x(t))=\left(\zeta_{1}\left(x_{1}(t)\right), \zeta_{2}\left(x_{2}(t)\right), \ldots, \zeta_{n}\left(x_{n}(t)\right)\right)^{T}$. Thereby, we have

$$
\zeta(x(t))=A(x(t)) B(x(t))-M x(t)
$$

And hence $\zeta(0)=A(0) B(0)-M \cdot 0=0$.

Next, we claim that System (12) with initial condition (9) is equivalent to

$$
\begin{aligned}
& x(t)=e^{-M t} \int_{0}^{t} e^{M r}\left\{-\zeta(x(r))+A(x(r)) \sum_{j=1}^{r^{*}} h_{j}(\omega(r))\left[C_{j} f(x(r))+D_{j} g(x(r-\tau(r)))+E_{j} \int_{r-\delta(r)}^{r} \eta(x(s)) d s\right]\right\} d r \\
& +e^{-M t} \phi(0)+e^{-M t} \sum_{0<t_{k}<t} e^{M t_{k}} \rho\left(x\left(t_{k}\right)\right), \quad t \geqslant 0
\end{aligned}
$$

with initial condition (9).

On one hand, we can prove that the solution of System Indeed, we get by (27) (27) with initial condition (9) is that of System (12) with initial condition (9).

$$
\begin{aligned}
e^{M t} x(t)= & \int_{0}^{t} e^{M r}\left\{-\zeta(x(r))+A(x(r)) \sum_{j=1}^{r^{*}} h_{j}(\omega(r))\left[C_{j} f(x(r))+D_{j} g(x(r-\tau(r)))+E_{j} \int_{r-\delta(r)}^{r} \eta(x(s)) d s\right]\right\} d r \\
& +\phi(0)+\sum_{0<t_{k}<t} e^{M t_{k}} \rho\left(x\left(t_{k}\right)\right), \quad t \geqslant 0,
\end{aligned}
$$

Differentiating both sides of (28) on $t$ results in that for $t \geqslant 0, t \neq t_{k}$,

$$
\begin{gathered}
M e^{M t} x(t)+e^{M t} \frac{d x(t)}{d t}=e^{M t}\{-\zeta(x(t))+A(x(t)) \\
\cdot \sum_{j=1}^{r^{*}} h_{j}(\omega(t))\left[C_{j} f(x(t))+D_{j} g(x(t-\tau(t)))\right.
\end{gathered}
$$

$$
\left.\left.+E_{j} \int_{t-\delta(t)}^{t} \eta(x(s)) d s\right]\right\}
$$

which generates the first equation of System (12).

Further, let $t \rightarrow t_{i}^{-}$in (27) produce $x\left(t_{i}^{-}\right)=\lim _{t \rightarrow t_{i}^{-}} x(t)=$ $x\left(t_{i}\right)$, and let $t \rightarrow t_{i}^{+}$in (27) come to $x\left(t_{i}^{+}\right)=\lim _{t \rightarrow t_{i}^{+}} x(t)=$ $x\left(t_{i}\right)+\rho\left(x\left(t_{i}\right)\right)$, which derives the second equation of (12). 
Thus, we have proved the above claim.

On the other hand, we claim that the solution of System (12) with initial condition (9) is that of System (27) with initial condition (9).

In fact, multiplying both sides of the first equation of System (12) with $e^{M t}$ results in

$$
e^{M t} \frac{d x(t)}{d t}+M e^{M t} x(t)=e^{M t}\{-\zeta(x(t))+A(x(t))
$$

$$
\begin{aligned}
& \cdot \sum_{j=1}^{r^{*}} h_{j}(\omega(t))\left[C_{j} f(x(t))+D_{j} g(x(t-\tau(t)))\right. \\
& \left.\left.+E_{j} \int_{t-\delta(t)}^{t} \eta(x(s)) d s\right]\right\},
\end{aligned}
$$

for all $t \geqslant 0, \quad t \neq t_{k}$. Moreover, integrating from $t_{k-1}+\varepsilon$ to $t \in\left(t_{k-1}, t_{k}\right)$ gives

$$
\begin{aligned}
& e^{M t} x(t)=e^{M\left(t_{k-1}+\varepsilon\right)} x\left(t_{k-1}+\varepsilon\right) \\
& \quad+\int_{t_{k-1}+\varepsilon}^{t} e^{M s}\left\{-\zeta(x(s))+A(x(s)) \sum_{j=1}^{r^{*}} h_{j}(\omega(s))\left[C_{j} f(x(s))+D_{j} g(x(s-\tau(s)))+E_{j} \int_{s-\delta(s)}^{s} \eta(x(r)) d r\right]\right\} d s,
\end{aligned}
$$

which yields, after letting $\varepsilon \rightarrow 0^{+}$,

$$
\begin{aligned}
& e^{M t} x(t)=e^{M\left(t_{k-1}\right)} x\left(t_{k-1}^{+}\right) \\
& \quad+\int_{t_{k-1}}^{t} e^{M s}\left\{-\zeta(x(s))+A(x(s)) \sum_{j=1}^{r^{*}} h_{j}(\omega(s))\left[C_{j} f(x(s))+D_{j} g(x(s-\tau(s)))+E_{j} \int_{s-\delta(s)}^{s} \eta(x(r)) d r\right]\right\} d s,
\end{aligned}
$$

for all $t \in\left(t_{k-1}, t_{k}\right)$.

$$
\begin{aligned}
& e^{M t_{k}-\varepsilon} x\left(t_{k}-\varepsilon\right)=e^{M t_{k-1}} x\left(t_{k-1}^{+}\right) \\
& \quad+\int_{t_{k-1}}^{t_{k}-\varepsilon} e^{M s}\left\{-\zeta(x(s))+A(x(s)) \sum_{j=1}^{r^{*}} h_{j}(\omega(s))\left[C_{j} f(x(s))+D_{j} g(x(s-\tau(s)))+E_{j} \int_{s-\delta(s)}^{s} \eta(x(r)) d r\right]\right\} d s,
\end{aligned}
$$

which yields by letting $\varepsilon \rightarrow 0^{+}$

$$
\begin{aligned}
& e^{M t_{k}} x\left(t_{k}\right)=e^{M t_{k-1}} x\left(t_{k-1}^{+}\right) \\
& \quad+\int_{t_{k-1}}^{t_{k}} e^{M s}\left\{-\zeta(x(s))+A(x(s)) \sum_{j=1}^{r^{*}} h_{j}(\omega(s))\left[C_{j} f(x(s))+D_{j} g(x(s-\tau(s)))+E_{j} \int_{s-\delta(s)}^{s} \eta(x(r)) d r\right]\right\} d s .
\end{aligned}
$$


Combining (32) and the above equation comes to

$$
\begin{aligned}
& e^{M t} x(t)=e^{M t_{k-1}} x\left(t_{k-1}^{+}\right) \\
& \quad+\int_{t_{k-1}}^{t} e^{M s}\left\{-\zeta(x(s))+A(x(s)) \sum_{j=1}^{r^{*}} h_{j}(\omega(s))\left[C_{j} f(x(s))+D_{j} g(x(s-\tau(s)))+E_{j} \int_{s-\delta(s)}^{s} \eta(x(r)) d r\right]\right\} d s \\
& \quad=e^{M t_{k-1}} x\left(t_{k-1}\right) \\
& +\int_{t_{k-1}}^{t} e^{M s}\left\{-\zeta(x(s))+A(x(s)) \sum_{j=1}^{r^{*}} h_{j}(\omega(s))\left[C_{j} f(x(s))+D_{j} g(x(s-\tau(s)))+E_{j} \int_{s-\delta(s)}^{s} \eta(x(r)) d r\right]\right\} d s \\
& +e^{M t_{k-1}} \rho\left(x\left(t_{k-1}\right)\right),
\end{aligned}
$$

for all $t \in\left(t_{k-1}, t_{k}\right], \quad k=1,2, \ldots$ Thereby, we have

$$
\begin{aligned}
& e^{M t_{k-1}} x\left(t_{k-1}\right)=e^{M t_{k-2}} x\left(t_{k-2}\right) \\
& +\int_{t_{k-2}}^{t_{k-1}} e^{M s}\left\{-\zeta(x(s))+A(x(s)) \sum_{j=1}^{r^{*}} h_{j}(\omega(s))\left[C_{j} f(x(s))+D_{j} g(x(s-\tau(s)))+E_{j} \int_{s-\delta(s)}^{s} \eta(x(r)) d r\right]\right\} d s \\
& +e^{M t_{k-2}} \rho\left(x\left(t_{k-2}\right)\right), \\
& +\int_{t_{1}}^{M t_{2}} x\left(t_{2}\right)=e^{M t_{1}} x\left(t_{1}\right) \\
& +e^{M t_{1}} \rho\left(x\left(t_{1}\right)\right), \\
& e^{M t_{1}} x\left(t_{1}\right)=\phi(0) \\
& +\int_{0}^{t_{1}} e^{M s}\left\{-\zeta(x(s))+A(x(s)) \sum_{j=1}^{r^{*}} h_{j}(\omega(s))\left[C_{j} f(x(s))+D_{j} g(x(s-\tau(s)))+E_{j} \int_{s-\delta(s)}^{s} \eta(x(r)) d r\right]\right\} d s .
\end{aligned}
$$

Synthesizing the above analysis derives System (27). Hence, we have proved that each solution of (12) with initial condition (9) is that of (27) with initial condition (9). Now, we have proved that System (12) with initial condition (9) is really equivalent to integral equations (27) with initial condition (9).

To apply the contraction mapping theorem, we firstly define the complete metric space $\mathscr{X}$ as follows.

Let $\mathscr{X}$ be the space consisting of functions $q(t)$ : $[-\tau, \infty) \rightarrow R^{n}$, satisfying the following:

(a) $q(t)$ is continuous on $t \in[0,+\infty) \backslash\left\{t_{k}\right\}_{k=1}^{\infty}$.

(b) $q(t)=\phi(t)$, for $t \in[-\tau, 0]$. (c) $\lim _{t \rightarrow t_{k}^{-}} q(t)=q\left(t_{k}\right)$ and $\lim _{t \rightarrow t_{k}^{+}} q(t)$ exists, for all $k=$ $1,2, \ldots$.

(d) $e^{\beta t} q(t) \rightarrow 0 \in R^{n}$ as $t \rightarrow+\infty$, where $\beta>0$ is a positive constant, satisfying $\beta<\lambda_{\min } M$.

It is not difficult to verify that the product space $\mathscr{X}$ is a complete metric space if it is equipped with the following metric:

$$
\operatorname{dist}(\bar{q}, \tilde{q})=\max _{i=1,2, \ldots, n}\left(\sup _{t \geqslant-\tau}\left|\bar{q}_{i}(t)-\tilde{q}_{i}(t)\right|\right)
$$

where $\bar{q}=\bar{q}(t)=\left(\bar{q}_{1}(t), \bar{q}_{2}(t), \ldots, \bar{q}_{n}(t)\right)^{T} \in \mathscr{X}$ and $\widetilde{q}=\widetilde{q}(t)=$ $\left(\widetilde{q}_{1}(t), \tilde{q}_{2}(t), \ldots, \tilde{q}_{n}(t)\right)^{T} \in \mathscr{X}$. 
Hence, we define the mapping $P$ as follows:

$$
\begin{aligned}
& P(x(t)) \\
& \quad=e^{-M t} \int_{0}^{t} e^{M r}\left\{-\zeta(x(r))+A(x(r)) \sum_{j=1}^{r^{*}} h_{j}(\omega(r))\left[C_{j} f(x(r))+D_{j} g(x(r-\tau(r)))+E_{j} \int_{r-\delta(r)}^{r} \eta(x(s)) d s\right]\right\} d r \\
& \quad+e^{-M t} \phi(0)+e^{-M t} \sum_{0<t_{k}<t} e^{M t_{k}} \rho\left(x\left(t_{k}\right)\right), \quad t \geqslant 0,
\end{aligned}
$$

and $P(x(s))=\phi(s)$ for all $s \in[-\tau, 0]$.

Below, we are to prove that $P$ is contraction mapping from $\mathscr{X}$ into $X$.

We may firstly prove $P(\mathscr{X}) \subset \mathscr{X}$. So we need to verify that $P(x)$ satisfies conditions (a)-(d) for all $x \in \mathscr{X}$.

Indeed, for $x(t) \in \mathscr{X}, \mathscr{P}(x(t))$ is continuous on $t \epsilon$ $[0,+\infty) \backslash\left\{t_{k}\right\}_{k=1}^{\infty}$ owing to (38), and $P(\cdot)$ satisfies condition (a). Further, the definition of $P$ implies that $P(\cdot)$ satisfies condition (b). Besides, we can derive from (38) that

$$
\begin{aligned}
P\left(x\left(t_{i}^{-}\right)\right) & =\lim _{\varepsilon \rightarrow 0^{+}} P\left(x\left(t_{i}-\varepsilon\right)\right)=P\left(x\left(t_{i}\right)\right), \\
P\left(x\left(t_{i}^{+}\right)\right) & =\lim _{\varepsilon \rightarrow 0^{+}} P\left(x\left(t_{i}+\varepsilon\right)\right) \\
& =P\left(x\left(t_{i}\right)\right)+\rho\left(x\left(t_{i}\right)\right),
\end{aligned}
$$

$$
i=1,2, \ldots,
$$

which comes to a conclusion that $P(\cdot)$ satisfies condition (c). Remark, the above convergence is under the metric of the metric space $\mathscr{X}$. Below, all the convergence is in this sense. No longer repeat.

Throughout this section, we assume that $\varepsilon$ is a sufficiently small positive real number.

Below, we need to prove that $P(\cdot)$ satisfies condition (d).

Indeed, for $x(t) \in \mathscr{X}$, we have

$$
\begin{aligned}
\left|e^{\beta t} P(x)\right| & =e^{\beta t}|P(x)| \\
& \leqslant U_{1}+U_{2}+U_{3}+U_{4}+U_{5}+U_{6},
\end{aligned}
$$

where

$$
\begin{aligned}
U_{1} & \triangleq e^{\beta t} e^{-M t} \int_{0}^{t} e^{M r}|-\zeta(x(r))| d r \\
& =e^{\beta t} e^{-M t} \int_{0}^{t} e^{M r}|\zeta(x(r))-\zeta(0)| d r \\
& \leqslant e^{\beta t} e^{-M t} \int_{0}^{t} e^{M r} L|x(r)| d r \\
& =e^{-(M-\beta I) t} \int_{0}^{t} e^{(M-\beta I) r} e^{\beta r} L|x(r)| d r .
\end{aligned}
$$

Owing to condition (d), for $x(t) \in \mathscr{X}, e^{\beta t} x(t) \rightarrow 0$ as $t \rightarrow$ $+\infty$. So, for any given $\varepsilon>0$, there exists sufficiently large $t_{*}>\tau>0$ such that

$$
\left|e^{\beta t} x(t)\right| \leqslant \varepsilon \xi, \quad \forall t \geqslant t_{*},
$$

where the fixed vector

$$
\xi \triangleq(1,1, \ldots, 1)^{T} \in R^{n}
$$

When $t>t_{*}$, we have

$$
\begin{aligned}
U_{1} & \leqslant e^{-(M-\beta I) t} \int_{0}^{t} e^{(M-\beta I) r} e^{\beta r} L|x(r)| d r \\
& \leqslant e^{-(M-\beta I) t}\left(\int_{0}^{t_{*}} e^{M r} L\left(\sum_{i=1}^{n} \sup _{r \in\left[-\tau, t_{*}\right]}\left|x_{i}(r)\right|\right) \xi d r\right. \\
& \left.+\int_{t_{*}}^{t} e^{(M-\beta I) r} L \varepsilon \xi d r\right) .
\end{aligned}
$$

It is obvious that

$$
e^{-(M-\beta I) t} \int_{0}^{t_{*}} e^{M r} L\left(\sum_{i=1}^{n} \sup _{r \in\left[-\tau, t_{*}\right]}\left|x_{i}(r)\right|\right) \xi d r \longrightarrow 0
$$

$$
t \longrightarrow+\infty \text {. }
$$

Besides,

$$
\begin{aligned}
& e^{-(M-\beta I) t} \int_{t_{*}}^{t} e^{(M-\beta I) r} d r \\
& \leqslant e^{-(M-\beta I) t} \operatorname{diag}\left(\frac{1}{m_{1}-\beta}, \ldots, \frac{1}{m_{n}-\beta}\right) e^{(M-\beta I) t} L \varepsilon \xi \\
& =\operatorname{diag}\left(\frac{1}{m_{1}-\beta}, \ldots, \frac{1}{m_{n}-\beta}\right) L \varepsilon \xi
\end{aligned}
$$

which together with arbitrariness implies that

$$
e^{-(M-\beta I) t} \int_{t_{*}}^{t} e^{(M-\beta I) r} d r \longrightarrow 0, \quad t \longrightarrow+\infty .
$$

Now we have actually proved that $U_{1} \rightarrow 0$ as $t \rightarrow+\infty$. 
Similarly, we have

$$
\begin{aligned}
U_{2} & \triangleq e^{\beta t} e^{-M t} \int_{0}^{t} e^{M r}|A(x(r))| \\
& \cdot\left|\sum_{j=1}^{r^{*}} h_{j}(\omega(r)) C_{j} f(x(r))\right| d r \\
& \leqslant e^{-(M-\beta I) t}\left(\int_{0}^{t_{*}} e^{M r} \bar{A} \sum_{j=1}^{r^{*}}\left|C_{j}\right| F|x(r)| d r\right. \\
& \left.+\int_{t_{*}}^{t} e^{(M-\beta I) r} \frac{r^{r^{*}}}{A} \sum_{j=1}\left|C_{j}\right| F\left(e^{\beta r}|x(r)|\right) d r\right) .
\end{aligned}
$$

Similarly, we use the methods employed in (44)-(47), obtain$\operatorname{ing} U_{2} \rightarrow 0$ as $t \rightarrow+\infty$.

Besides,

$$
\begin{aligned}
& U_{3} \triangleq e^{\beta t} e^{-M t} \int_{0}^{t} e^{M r}|A(x(r))| \sum_{j=1}^{r^{*}} h_{j}(\omega(r)) \\
& \cdot D_{j} g(x(r-\tau(r))) \mid d r \\
& \leqslant e^{-(M-\beta I) t}\left(\int_{0}^{t_{*}+\tau} e^{M r} \bar{A} \sum_{j=1}^{r^{*}}\left|D_{j}\right|\right. \\
& \cdot G|x(r-\tau(r))| d r \\
& +\int_{t_{*}+\tau}^{t} e^{(M-\beta I) r} e^{\beta \tau(r)} \frac{{ }^{2}}{A} \sum_{j=1}^{r^{*}}\left|D_{j}\right| \\
& \left.+e^{\beta \tau} \int_{t_{*}+\tau}^{t} e^{(M-\beta I) r} \bar{A} \sum_{j=1}^{r^{*}}\left|D_{j}\right| G \varepsilon \xi d r\right) \\
& \left.+e^{\beta(r-\tau(r))}|x(r-\tau(r))| d r\right) \\
& \leqslant e^{-(M-\beta I) t}\left(\int_{0}^{t_{*}+\tau} e^{M r} \bar{A} \sum_{j=1}^{r^{*}}\left|D_{j}\right| G\right.
\end{aligned}
$$

Besides,

$$
\begin{aligned}
& e^{-(M-\beta I) t} \int_{t_{*}+\tau}^{t} e^{M r} \bar{A} \sum_{j=1}^{r^{*}}\left|E_{j}\right| \int_{r-\tau}^{r} Q|x(s)| d s d r \\
& \leqslant e^{-(M-\beta I) t} \int_{t_{*}+\tau}^{t} e^{M r} e^{-\beta(r-\tau)} \frac{r^{*}}{A} \sum_{j=1}^{*}\left|E_{j}\right| \\
& \cdot \int_{r-\tau}^{r} Q e^{\beta s}|x(s)| d s d r \leqslant \varepsilon \\
& \cdot \operatorname{diag}\left(\frac{1}{m_{1}-\beta}, \ldots, \frac{1}{m_{n}-\beta}\right) e^{\beta \tau} \frac{r^{*}}{A} \sum_{j=1}^{r^{*}}\left|E_{j}\right| \tau Q \xi .
\end{aligned}
$$

Similarly, we use the methods employed in (44)-(47), obtain$\operatorname{ing} U_{4} \rightarrow 0$ as $t \rightarrow+\infty$.

Obviously, $U_{5} \triangleq\left|e^{\beta t} e^{-M t} \phi(0)\right| \rightarrow 0$ as $t \rightarrow+\infty$.

Next,

It is clear that

$$
\begin{aligned}
& e^{-(M-\beta I) t} \int_{0}^{t_{*}+\tau} e^{M r} \bar{A} \sum_{j=1}^{r^{*}}\left|E_{j}\right| \tau Q \\
& \cdot \sum_{i=1}^{n} \sup _{s \in\left[-\tau, t_{*}+\tau\right]}\left|x_{i}(s)\right| \xi d r \longrightarrow 0 \text { as } t \longrightarrow+\infty .
\end{aligned}
$$


Below, we assume that $t_{m}<t_{*} \leqslant t_{m+1}$ and $t_{j}<t \leqslant t_{j+1}$ with $j=0,1,2, \ldots$. Here, $t_{0}=0$.

$$
\begin{aligned}
U_{6} & \triangleq e^{\beta t} e^{-M t} \sum_{0<t_{k}<t} e^{M t_{k}}\left|\rho\left(x\left(t_{k}\right)\right)\right| \\
& \leqslant e^{-(M-\beta I) t}\left(\sum_{0<t_{k} \leqslant t_{*}} e^{M t_{k}} H\left|x\left(t_{k}\right)\right|\right. \\
& \left.+\sum_{t_{*}<t_{k}<t} e^{M t_{k}} H\left|x\left(t_{k}\right)\right|\right) .
\end{aligned}
$$

Obviously,

$$
\begin{aligned}
e^{-(M-\beta I) t} \sum_{0<t_{k} \leqslant t_{*}} e^{M t_{k}} H\left|x\left(t_{k}\right)\right| \\
=e^{-(M-\beta I) t} \sum_{t_{1} \leqslant t_{k} \leqslant t_{m+1}} e^{M t_{k}} H\left|x\left(t_{k}\right)\right| \longrightarrow 0,
\end{aligned}
$$

$t \longrightarrow+\infty$.

Next,

$$
\begin{aligned}
& e^{-(M-\beta I) t} \sum_{t_{*}<t_{k}<t} e^{M t_{k}} H\left|x\left(t_{k}\right)\right| \\
& \leqslant e^{-(M-\beta I) t} \sum_{t_{m+1} \leqslant t_{k} \leqslant t_{j}} e^{(M-\beta I) t_{k}} H \varepsilon \xi \leqslant e^{-(M-\beta I) t}
\end{aligned}
$$

$$
\begin{aligned}
& \cdot \frac{1}{\mu}\left(\mu e^{(M-\beta I) t_{j}} H \varepsilon \xi\right. \\
+ & \left.\sum_{t_{m+1} \leqslant t_{k} \leqslant t_{j-1}}\left(t_{k+1}-t_{k}\right) e^{(M-\beta I) t_{k}} H \varepsilon \xi\right) \leqslant e^{-(M-\beta I) t} \\
& \frac{1}{\mu}\left(\mu e^{(M-\beta I) t_{j}} H \varepsilon \xi+\int_{t^{*}}^{t} e^{(M-\beta I) s} d s H \varepsilon \xi\right) \leqslant \varepsilon \\
& \cdot \frac{1}{\mu}\left(\mu+\operatorname{diag}\left(\frac{1}{m_{1}-\beta}, \ldots, \frac{1}{m_{n}-\beta}\right)\right) H \xi
\end{aligned}
$$

which together with the arbitrariness of $\varepsilon$ implies

$$
e^{-(M-\beta I) t} \sum_{t_{*}<t_{k}<t} e^{M t_{k}} H\left|x\left(t_{k}\right)\right| \longrightarrow 0, \quad t \longrightarrow+\infty
$$

So we have proved that $U_{6} \rightarrow 0$ as $t \rightarrow+\infty$.

Synthesizing the above analysis results in $e^{\beta t} P(x(t)) \rightarrow 0$ as $t \rightarrow+\infty$, for all $x(t) \in \mathscr{X}$. Thus, $P(\cdot)$ satisfies all conditions (a)-(d), which derives $P(\mathscr{X}) \subset \mathscr{X}$.

Finally, we claim that $P: \mathscr{X} \rightarrow \mathscr{X}$ is contraction mapping. Indeed, for any $x, y \in \mathscr{X}$,

$$
\begin{aligned}
& |P(x)-P(y)| \\
& \quad \leqslant e^{-M t} \mid \int_{0}^{t} e^{M r}\left\{-\zeta(x(r))+A(x(r)) \sum_{j=1}^{r^{*}} h_{j}(\omega(r))\left[C_{j} f(x(r))+D_{j} g(x(r-\tau(r)))+E_{j} \int_{r-\delta(r)}^{r} \eta(x(s)) d s\right]\right\} d r \\
& \left.-\int_{0}^{t} e^{M r}\left\{-\zeta(y(r))+A(y(r)) \sum_{j=1}^{r^{*}} h_{j}(\omega(r))\left[C_{j} f(y(r))+D_{j} g(y(r-\tau(r)))+E_{j} \int_{r-\delta(r)}^{r} \eta(y(s)) d s\right]\right\} d r \mid\right\} \mid \leqslant K_{1}+K_{2}+K_{3}+K_{4}+K_{5}, \\
& +e^{-M t}\left|\sum_{0<t_{k}<t} e^{M t_{k}} \rho\left(x\left(t_{k}\right)\right)-\sum_{0<t_{k}<t} e^{M t_{k}} \rho\left(y\left(t_{k}\right)\right)\right|
\end{aligned}
$$

where

$$
\begin{aligned}
K_{1} & \triangleq e^{-M t} \int_{0}^{t} e^{M r}|\zeta(x(r))-\zeta(y(r))| d r \\
& \leqslant e^{-M t} \int_{0}^{t} e^{M r} L|x(r)-y(r)| d r \\
& \leqslant e^{-M t} \int_{0}^{t} e^{M r} L d r \operatorname{dist}(x, y) \xi \leqslant e^{-M t} M^{-1} e^{M t} L \\
\cdot & \operatorname{dist}(x, y) \xi=M^{-1} L \operatorname{dist}(x, y) \xi \\
K_{2} & \triangleq e^{-M t} \int_{0}^{t} e^{M r} \mid A(x(r)) \sum_{j=1}^{r^{*}} h_{j}(\omega(r)) C_{j} f(x(r))
\end{aligned}
$$

$$
\begin{aligned}
& -A(y(r)) \sum_{j=1}^{r^{*}} h_{j}(\omega(r)) C_{j} f(y(r)) \mid d r \\
& \leqslant e^{-M t} \int_{0}^{t} e^{M r}\left(|A(x(r))| \sum_{j=1}^{r^{*}}\left|C_{j}\right|\right. \\
& \cdot|f(x(r))-f(y(r))|+|A(x(r))-A(y(r))| \\
& \left.\cdot \sum_{j=1}^{r^{*}}\left|C_{j}\right||f(y(r))|\right) d r \\
& \leqslant e^{-M t} \int_{0}^{t} e^{M r}\left(\bar{A} \sum_{j=1}^{r^{*}}\left|C_{j}\right| F|x(r)-y(r)|+\mathscr{A}\right.
\end{aligned}
$$


Journal of Function Spaces

11

$$
\begin{aligned}
& \cdot \operatorname{diag}\left(\left|x_{1}(r)-y_{1}(r)\right|, \ldots,\left|x_{n}(r)-y_{n}(r)\right|\right) \sum_{j=1}^{r^{*}}\left|C_{j}\right| \\
& \leqslant e^{-M t} M^{-1} e^{M t}\left(\bar{A} \sum_{j=1}^{r^{*}}\left|D_{j}\right| G \operatorname{dist}(x, y) \xi+\mathscr{A}\right. \\
& \left.\cdot F^{*} \xi\right) d r \leqslant e^{-M t} \int_{0}^{t} e^{M r} \bar{A} \sum_{j=1}^{r^{*}}\left|C_{j}\right| F \operatorname{dist}(x, y) \\
& \left.\cdot \operatorname{dist}(x, y) I \sum_{j=1}^{r^{*}}\left|D_{j}\right| G^{*} \xi\right)=M^{-1}\left(\bar{A} \sum_{j=1}^{r^{*}}\left|D_{j}\right| G\right. \\
& \xi \xi d r+e^{-M t} \int_{0}^{t} e^{M r} \mathscr{A} \\
& \left.+G^{*} \mathscr{A} \sum_{j=1}^{r^{*}}\left|D_{j}\right|\right) \operatorname{dist}(x, y) \xi \\
& \cdot \operatorname{diag}\left(\left|x_{1}(r)-y_{1}(r)\right|, \ldots,\left|x_{n}(r)-y_{n}(r)\right|\right) \sum_{j=1}^{r^{*}}\left|C_{j}\right| \\
& K_{4} \triangleq e^{-M t} \int_{0}^{t} e^{M r} \mid A(x(r)) \sum_{j=1}^{r^{*}} h_{j}(\omega(r)) \\
& \cdot F^{*} \xi d r \leqslant e^{-M t} \int_{0}^{t} e^{M r} d r \bar{A} \sum_{j=1}^{r^{*}}\left|C_{j}\right| F \operatorname{dist}(x, y) \xi \\
& \cdot E_{j} \int_{r-\delta(r)}^{r} \eta(x(s)) d s-A(y(r)) \sum_{j=1}^{r^{*}} h_{j}(\omega(r)) E_{j} \\
& +e^{-M t} \int_{0}^{t} e^{M r} \mathscr{A} \operatorname{dist}(x, y) I \sum_{j=1}^{r^{*}}\left|C_{j}\right| F^{*} \xi d r \\
& \left.\cdot \int_{r-\delta(r)}^{r} \eta(y(s)) d s\right) \mid d r \\
& \leqslant M^{-1}\left(\bar{A} \sum_{j=1}^{r^{*}}\left|C_{j}\right| F+F^{*} \mathscr{A} \sum_{j=1}^{r^{*}}\left|C_{j}\right|\right) \operatorname{dist}(x, y) \xi \text {, } \\
& \leqslant e^{-M t} \int_{0}^{t} e^{M r}\left(|A(x(r))| \sum_{j=1}^{r^{*}}\left|E_{j}\right|\right. \\
& K_{3} \triangleq e^{-M t} \int_{0}^{t} e^{M r} \mid A(x(r)) \sum_{j=1}^{r^{*}} h_{j}(\omega(r)) \\
& \cdot \int_{r-\delta(r)}^{r}|\eta(x(s))-\eta(y(s))| d s+\mid A(x(r)) \\
& \cdot D_{j} g(x(r-\tau(r)))-A(y(r)) \sum_{j=1}^{r^{*}} h_{j}(\omega(r)) \\
& \left.-A(y(r))\left|\sum_{j=1}^{r^{*}}\right| E_{j}\left|\int_{r-\delta(r)}^{r}\right| \eta(y(s)) \mid d s\right) d r \\
& \cdot D_{j} g(y(r-\tau(r))) \mid d r \\
& \leqslant e^{-M t} \int_{0}^{t} e^{M r}\left(\bar{A} \sum_{j=1}^{r^{*}}\left|E_{j}\right| \int_{r-\tau}^{r} Q|x(s)-y(s)| d s\right. \\
& \leqslant e^{-M t} \int_{0}^{t} e^{M r}\left(|A(x(r))| \sum_{j=1}^{r^{*}}\left|D_{j}\right|\right. \\
& +\mathscr{A} \operatorname{diag}\left(\left|x_{1}(r)-y_{1}(r)\right|, \ldots,\left|x_{n}(r)-y_{n}(r)\right|\right) \\
& \left.\cdot \sum_{j=1}^{r^{*}}\left|E_{j}\right| \int_{r-\tau}^{r} Q^{*} \xi d s\right) d r \leqslant M^{-1} \tau\left(\bar{A} \sum_{j=1}^{r^{*}}\left|E_{j}\right|\right. \\
& \left.\cdot Q+Q^{*} \mathscr{A} \sum_{j=1}^{r^{*}}\left|E_{j}\right|\right) \operatorname{dist}(x, y) \xi \\
& \leqslant e^{-M t} \int_{0}^{t} e^{M r}\left(\bar{A} \sum_{j=1}^{r^{*}}\left|D_{j}\right|\right. \\
& K_{5} \triangleq e^{-M t}\left|\sum_{0<t_{k}<t} e^{M t_{k}} \rho\left(x\left(t_{k}\right)\right)-\sum_{0<t_{k}<t} e^{M t_{k}} \rho y\left(t_{k}\right)\right| \\
& \cdot G|x(r-\tau(r))-y(r-\tau(r))|+\mathscr{A} \\
& \cdot \operatorname{diag}\left(\left|x_{1}(r)-y_{1}(r)\right|, \ldots,\left|x_{n}(r)-y_{n}(r)\right|\right) \sum_{j=1}^{r^{*}}\left|D_{j}\right| \\
& \leqslant e^{-M t} \sum_{0<t_{k}<t} e^{M t_{k}}\left|\rho\left(x\left(t_{k}\right)\right)-\rho y\left(t_{k}\right)\right| \\
& \leqslant e^{-M t} \sum_{0<t_{k}<t} e^{M t_{k}} H\left|x\left(t_{k}\right)-y\left(t_{k}\right)\right| \leqslant \operatorname{dist}(x, y) \\
& \left.\cdot G^{*} \xi\right) d r \leqslant e^{-M t} \int_{0}^{t} e^{M r}\left(\bar{A} \sum_{j=1}^{r^{*}}\left|D_{j}\right| G\right. \\
& \cdot e^{-M t} \sum_{0<t_{k}<t} e^{M t_{k}} H \xi \leqslant \frac{1}{\mu} \operatorname{dist}(x, y) e^{-M t} \\
& \left.\cdot \operatorname{dist}(x, y) \xi+\mathscr{A} \operatorname{dist}(x, y) I \sum_{j=1}^{r^{*}}\left|D_{j}\right| G^{*} \xi\right) d r \\
& \text {. } \sum_{t_{1} \leqslant t_{k} \leqslant t_{j-1}}\left[\left(t_{k+1}-t_{k}\right) e^{M t_{k}}+\mu e^{M t_{j}}\right] H \xi \leqslant \frac{1}{\mu} \operatorname{dist}(x,
\end{aligned}
$$




$$
\begin{aligned}
& y) e^{-M t}\left(\int_{0}^{t} e^{M s} d s+\mu e^{M t}\right) H \xi \leqslant\left(\frac{1}{\mu} M^{-1} H\right. \\
& +H) \operatorname{dist}(x, y) \xi
\end{aligned}
$$

where we assume $t_{j}<t \leqslant t_{j+1}$ with $j=0,1,2, \ldots$. Here, $t_{0}=0$.

Combining the above five inequalities and (24) produces

$$
\begin{aligned}
& |P(x(t))-P(y(t))| \leqslant\left[M^{-1} L\right. \\
& \quad+M^{-1}\left(\bar{A}|C| F+F^{*} \mathscr{A}|C|\right) \\
& \quad+M^{-1}\left(\bar{A}|D| G+G^{*} \mathscr{A}|D|\right) \\
& \quad+M^{-1} \tau\left(\bar{A}|E| Q+Q^{*} \mathscr{A}|E|\right) \\
& \left.\quad+\left(\frac{1}{\mu} M^{-1} H+H\right)\right] \operatorname{dist}(x, y) \xi \\
& \quad<\alpha \operatorname{dist}(x, y) \xi,
\end{aligned}
$$

and hence

$$
\operatorname{dist}(P(x), P(x)) \leqslant \alpha \operatorname{dist}(x, y) \text {. }
$$

Therefore, $P: \mathscr{X} \rightarrow \mathscr{X}$ is contraction mapping such that there exists the fixed point $x(t)$ of $P$ in $\mathscr{X}$, which implies that $x(t)$ is the solution for the impulsive fuzzy CGNNs (12) with initial condition (9), satisfying $e^{\beta t}\|x(t)\| \rightarrow 0$ as $t \rightarrow+\infty$. So the proof is completed.

Remark 9. From the proof, we know that the formulated contraction mapping is different from that of existing literature involved in the fixed point technique and CGNNs models. One can also understand from Remark 1 that we proposed in this paper a really feasible new condition on amplification function $a_{i}$ and behavior function $b_{i}$. This implies that our result and methods are novelty versus [18, Theorem 4]. Besides, our conditions and result are also different from those of existing literature [17, 19-22] (see below "Table 1" and "Remark 13").

In case of ignoring fuzzy factors and distributed delay (letting $E=0$ ) in (12), we can get the following impulsive CGNNs with discrete delay

$$
\begin{aligned}
& \frac{d x(t)}{d t}=-A(x(t)) \\
& \cdot[B(x(t))-C f(x(t))-D g(x(t-\tau(t)))], \\
& \quad t \geqslant 0, t \neq t_{k}, \\
& x\left(t_{k}^{+}\right)-x\left(t_{k}^{-}\right)=\rho\left(x\left(t_{k}\right)\right), \quad k=1,2, \ldots
\end{aligned}
$$

Corollary 10. Impulsive CGNNs (61) with initial condition (9) are global exponential stability if there is a positive number $\alpha<$ 1, satisfying the following LMI condition:

$$
\begin{aligned}
& L+\bar{A}|C| F+F^{*} \mathscr{A}|C|+\bar{A}|D| G+G^{*} \mathscr{A}|D|+\frac{1}{\mu} H \\
& +M H-\alpha M<0,
\end{aligned}
$$

where $\mu=\inf _{k=1,2, \ldots}\left(t_{k+1}-t_{k}\right)>0$.

Remark 11. As a corollary of our main result, the conditions and conclusion are better than the main result of [18] (see Remark 1).

\section{Numerical Example}

Example 1. Consider the T-S fuzzy impulsive discrete and distributed delays CGNNs model as follows.

Fuzzy Rule 1. IF $\omega_{1}(t)$ is $1 / e^{-2 \omega_{1}(t)}$, THEN

$$
\begin{aligned}
& \frac{d x(t)}{d t}=-A(x(t))\left[B(x(t))-C_{1} f(x(t))\right. \\
& \left.-D_{1} g(x(t-\tau(t)))-E_{1} \int_{t-\delta(t)}^{t} \eta(x(s)) d s\right], \\
& t \geqslant 0, t \neq t_{k}, \\
& x\left(t_{k}^{+}\right)-x\left(t_{k}^{-}\right)=\rho\left(x\left(t_{k}\right)\right), \quad k=1,2, \ldots, \\
& x(s)=\phi(s), \quad s \in[-\tau, 0] .
\end{aligned}
$$

Fuzzy Rule 2. IF $\omega_{2}(t)$ is $1-1 / e^{-2 \omega_{1}(t)}$, THEN

$$
\begin{aligned}
& \frac{d x(t)}{d t}=-A(x(t))\left[B(x(t))-C_{2} f(x(t))\right. \\
& \left.-D_{2} g(x(t-\tau(t)))-E_{2} \int_{t-\delta(t)}^{t} \eta(x(s)) d s\right], \\
& t \geqslant 0, t \neq t_{k}, \\
& x\left(t_{k}^{+}\right)-x\left(t_{k}^{-}\right)=\rho\left(x\left(t_{k}\right)\right), \quad k=1,2, \ldots, \\
& x(s)=\phi(s), \quad s \in[-\tau, 0],
\end{aligned}
$$

where $r^{*}=2, F^{*}=0.2897, G^{*}=0.2679, Q^{*}=0.2102$,

$$
\begin{aligned}
& D_{1}=\left(\begin{array}{cc}
0.1213 & 0.011 \\
0 & 0.1312
\end{array}\right), \\
& E_{1}=\left(\begin{array}{cc}
0.1511 & 0.0101 \\
0 & 0.1887
\end{array}\right), \\
& D_{2}=\left(\begin{array}{cc}
0.1812 & 0 \\
0.002 & 0.1002
\end{array}\right),
\end{aligned}
$$




$$
\begin{aligned}
& E_{2}=\left(\begin{array}{cc}
0.1811 & 0 \\
0.0210 & 0.1077
\end{array}\right), \\
& \bar{A}=\left(\begin{array}{cc}
0.2111 & 0 \\
0 & 0.1192
\end{array}\right), \\
& C_{1}=\left(\begin{array}{cc}
0.2113 & 0.0111 \\
0 & 0.299
\end{array}\right) \text {, } \\
& C_{2}=\left(\begin{array}{cc}
0.1811 & 0 \\
0.0021 & 0.1102
\end{array}\right) \text {, } \\
& G=\left(\begin{array}{cc}
0.1011 & 0 \\
0 & 0.2102
\end{array}\right), \\
& L=\left(\begin{array}{cc}
0.1318 & 0 \\
0 & 0.1201
\end{array}\right) \text {, } \\
& Q=\left(\begin{array}{cc}
0.1002 & 0 \\
0 & 0.2001
\end{array}\right), \\
& \mathscr{A}=\left(\begin{array}{cc}
0.1812 & 0 \\
0 & 0.1901
\end{array}\right) \text {, } \\
& F=\left(\begin{array}{cc}
0.1027 & 0 \\
0 & 0.2103
\end{array}\right) \text {, } \\
& H=\left(\begin{array}{cc}
0.4586 & 0 \\
0 & 0.4386
\end{array}\right) \text {, } \\
& M=\left(\begin{array}{cc}
2.9013 & 0 \\
0 & 2.8131
\end{array}\right)
\end{aligned}
$$

Let $\mu=0.5, \tau=11.7$, then we can use MATLAB LMI toolbox to solve the LMI condition (24), extracting the feasible datum

$$
\alpha=0.9981,
$$

which means $0<\alpha<1$. Thereby, we can conclude from Theorem 8 that the impulsive fuzzy dynamic equations are globally exponentially stable.

Remark 12. From Example 1, the allowable upper bound of time-delays $\tau=11.7$ and the pulse interval $\mu=0.5$ indicate that the range of feasibility is extensive and broad.

Table 1 shows the comparison of our Theorem 8 and Corollary 10 with other criteria of CGNNs (derived by fixed point theorems) about the behavior function $b_{j}$ and programmability for computer software.

Remark 13. From Table 1, we know that the conditions of our behavior function $b_{j}$ are weaker than those of existing results published from 2007 to 2016. Moreover, our criterion is LMIbased, which is programmable for computer LMI toolbox. In addition, our Corollary 10 is better than [18, Theorem 4], the main result of [18] (see Remark 4 for details).

\section{Conclusions}

In many existing literatures related to Cohen-Grossberg Neural Networks, various fixed point theorems were applied to derive the stability criteria by imitating System (1). However, in this paper, the authors proposed a new condition on behavior functions and formulate new contraction mapping to derive a new LMI-based exponential stability. To a certain extent, this paper improves the previous related results (see Table 1, Remarks 4 and 13). Moreover, numerical example is presented to illustrate the feasibility and effectiveness of the proposed methods.

\section{Competing Interests}

The authors declare that they have no competing interests.

\section{Authors' Contributions}

All authors typed, read, and approved the final manuscript.

\section{Acknowledgments}

This work was supported by National Basic Research Program of China (2010CB732501), Scientific Research Fund of Science Technology Department of Sichuan Province (2012JY010), Sichuan Educational Committee Science Foundation (08ZB002, 12ZB349, and 14ZA0274), and the Initial Founding of Scientific Research for Chengdu Normal University Introduction of Talents.

\section{References}

[1] M. Cohen and S. Grossberg, "Absolute stability and global pattern formation and parallel memory storage by competitive neural networks," IEEE Transactions on Systems, Man, and Cybernetics, vol. 13, pp. 815-826, 1983.

[2] A. Zada, S. Faisal, and Y. Li, "On the Hyers-Ulam stability of first-order impulsive delay differential equations," Journal of Function Spaces, vol. 2016, Article ID 8164978, 6 pages, 2016.

[3] X. Li, R. Rakkiyappan, and G. Velmurugan, "Dissipativity analysis of memristor-based complex-valued neural networks with time-varying delays," Information Sciences, vol. 294, pp. 645-665, 2015.

[4] R. Rao, "Delay-dependent exponential stability for nonlinear reaction-diffusion uncertain Cohen-Grossberg neural networks with partially known transition rates via Hardy-Poincaré inequality," Chinese Annals of Mathematics Series B, vol. 35, no. 4, pp. 575-598, 2014.

[5] X. Li and X. Fu, "Global asymptotic stability of stochastic Cohen-Grossberg-type BAM neural networks with mixed delays: an LMI approach," Journal of Computational and Applied Mathematics, vol. 235, no. 12, pp. 3385-3394, 2011.

[6] X. Li, M. Bohner, and C.-K. Wang, "Impulsive differential equations: periodic solutions and applications," Automatica, vol. 52, pp. 173-178, 2015.

[7] R. Rao, S. Zhong, and X. Wang, "Stochastic stability criteria with LMI conditions for Markovian jumping impulsive BAM neural networks with mode-dependent time-varying delays and nonlinear reaction-diffusion," Communications in Nonlinear 
Science and Numerical Simulation, vol. 19, no. 1, pp. 258-273, 2014.

[8] S. Long and S. Zhong, "Improved results for stochastic stabilization of a class of discretetime singular Markovian jump systems with time-varying delay," Nonlinear Analysis: Hybrid Systems, vol. 23, pp. 11-26, 2017.

[9] Q. Song, Z. Zhao, and Y. Liu, "Impulsive effects on stability of discrete-time complex-valued neural networks with both discrete and distributed time-varying delays," Neurocomputing, vol. 168, pp. 1044-1050, 2015.

[10] X. Li and J. Wu, "Stability of nonlinear differential systems with state-dependent delayed impulses," Automatica, vol. 64, pp. 6369, 2016.

[11] J. Xiao, S. Zhong, and Y. Li, "Improved passivity criteria for memristive neural networks with interval multiple timevarying delays," Neurocomputing, vol. 171, pp. 1414-1430, 2016.

[12] X. Li and S. Song, "Stabilization of Delay Systems: delaydependent Impulsive Control," IEEE Transactions on Automatic Control, vol. 62, no. 1, pp. 406-411, 2017.

[13] Q. Song, H. Yan, Z. Zhao, and Y. Liu, "Global exponential stability of complex-valued neural networks with both timevarying delays and impulsive effects," Neural Networks, vol. 79, pp. 108-116, 2016.

[14] H. Zhu and C. Feng, "Existence and global uniform asymptotic stability of pseudo almost periodic solutions for CohenGrossberg neural networks with discrete and distributed delays," Mathematical Problems in Engineering, vol. 2014, Article ID 968404, 10 pages, 2014.

[15] Z. Mao and H. Zhao, "Dynamical analysis of Cohen-Grossberg neural networks with distributed delays," Physics Letters A, vol. 364, no. 1, pp. 38-47, 2007.

[16] X. Yang, C. Huang, D. Zhang, and Y. Long, "Dynamics of Cohen-Grossberg neural networks with mixed delays and impulses," Abstract and Applied Analysis, vol. 2008, Article ID 432341, 14 pages, 2008.

[17] Z. Mao, H. Zhao, and X. Wang, "Dynamics of Cohen-Grossberg neural networks with variable and distributed delays," Physica D, vol. 234, no. 1, pp. 11-22, 2007.

[18] X. Wang, R. Rao, and S. Zhong, "LMI-based stability criterion for impulsive CGNNs via fixed point theory," Mathematical Problems in Engineering, vol. 2015, Article ID 281681, 10 pages, 2015.

[19] H. Xiang and J. Cao, "Almost periodic solution of CohenGrossberg neural networks with bounded and unbounded delays," Nonlinear Analysis: Real World Applications, vol. 10, no. 4, pp. 2407-2419, 2009.

[20] H. Zhao, L. Chen, and Z. Mao, "Existence and stability of almost periodic solution for Cohen-Grossberg neural networks with variable coefficients," Nonlinear Analysis: Real World Applications, vol. 9, no. 2, pp. 663-673, 2008.

[21] Y. Li, T. Zhang, and Z. Xing, "The existence of nonzero almost periodic solution for Cohen-Grossberg neural networks with continuously distributed delays and impulses," Neurocomputing, vol. 73, no. 16-18, pp. 3105-3113, 2010.

[22] B. Li and Q. Song, "Some new results on periodic solution of Cohen-Grossbergneural network with impulses," Neurocomputing, vol. 177, pp. 401-408, 2016.

[23] R. Rao, S. Zhong, and Z. Pu, "On the role of diffusion factors in stability analysis for $\mathrm{p}$-Laplace dynamical equations involved to BAM Cohen-Grossberg neural network," Neurocomputing, vol. 223, pp. 54-62, 2017.
[24] T. Liang, Y. Yang, Y. Liu, and L. Li, "Existence and global exponential stability of almost periodic solutions to CohenGrossberg neural networks with distributed delays on time scales," Neurocomputing, vol. 123, pp. 207-215, 2014.

[25] Y. Yang, T. Liang, and X. Xu, "Almost sure exponential stability of stochastic Cohen-Grossberg neural networks with continuous distributed delays of neutral type," Optik, vol. 126, no. 23, pp. 4628-4635, 2015.

[26] J. J. Oliveira, "Global stability of a Cohen-Grossberg neural network with both time-varying and continuous distributed delays," Nonlinear Analysis: Real World Applications, vol. 12, no. 5, pp. 2861-2870, 2011.

[27] K. Li, L. Zhang, X. Zhang, and Z. Li, "Stability in impulsive Cohen-Grossberg-type BAM neural networks with distributed delays," Applied Mathematics and Computation, vol. 215, no. 11, pp. 3970-3984, 2010.

[28] T. Li, S.-M. Fei, Y.-Q. Guo, and Q. Zhu, "Stability analysis on Cohen-Grossberg neural networks with both time-varying and continuously distributed delays," Nonlinear Analysis: Real World Applications, vol. 10, no. 4, pp. 2600-2612, 2009.

[29] Q. Song, Z. Wang, and J. Liang, "Analysis on passivity and passification of T-S fuzzy systems with time-varying delays," Journal of Intelligent and Fuzzy Systems, vol. 24, no. 1, pp. 21-30, 2013.

[30] P. Balasubramaniam, V. Vembarasan, and R. Rakkiyappan, "Delay-dependent robust exponential state estimation of Markovian jumping fuzzy Hopfield neural networks with mixed random time-varying delays," Communications in Nonlinear Science and Numerical Simulation, vol. 16, no. 4, pp. 2109-2129, 2011.

[31] S. Muralisankar, N. Gopalakrishnan, and P. Balasubramaniam, "An LMI approach for global robust dissipativity analysis of T-S fuzzy neural networks with interval time-varying delays," Expert Systems with Applications, vol. 39, no. 3, pp. 3345-3355, 2012.

[32] D. R. Smart, Fixed Point Theorems, Cambridge University Press, Cambridgem, UK, 1980. 


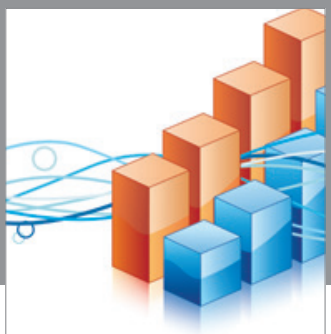

Advances in

Operations Research

vatem alat4

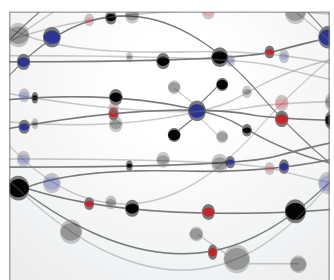

\section{The Scientific} World Journal
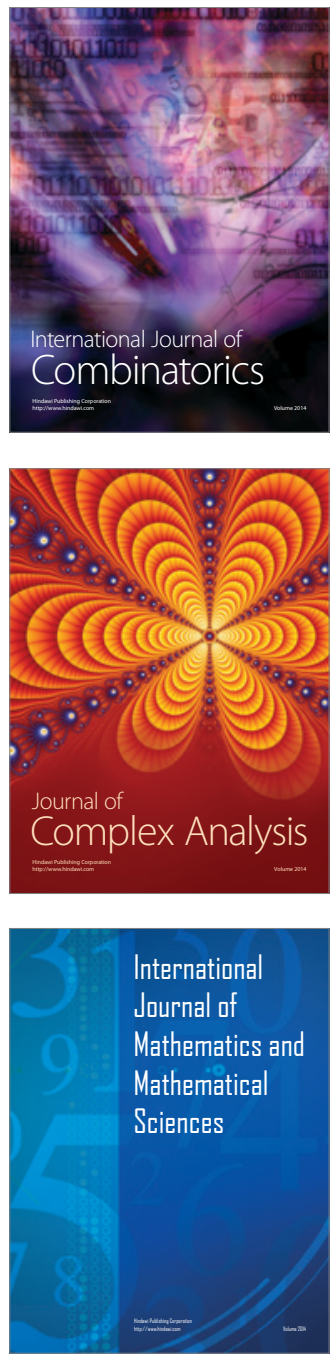
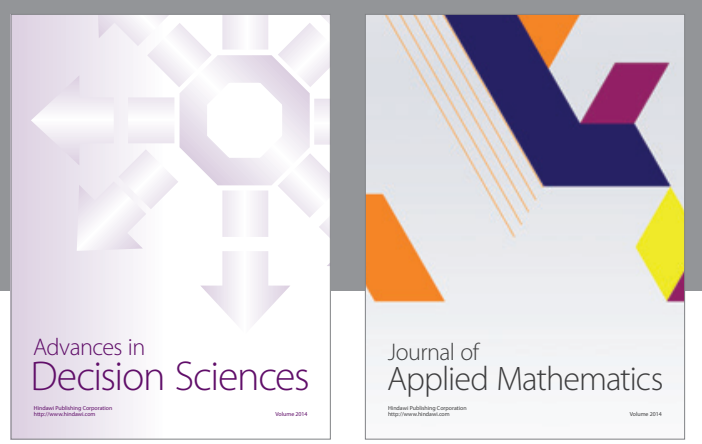

Algebra

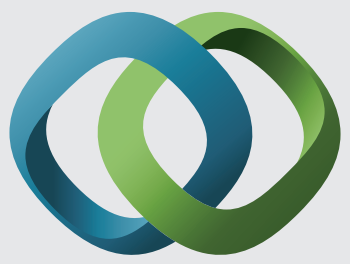

\section{Hindawi}

Submit your manuscripts at

http://www.hindawi.com
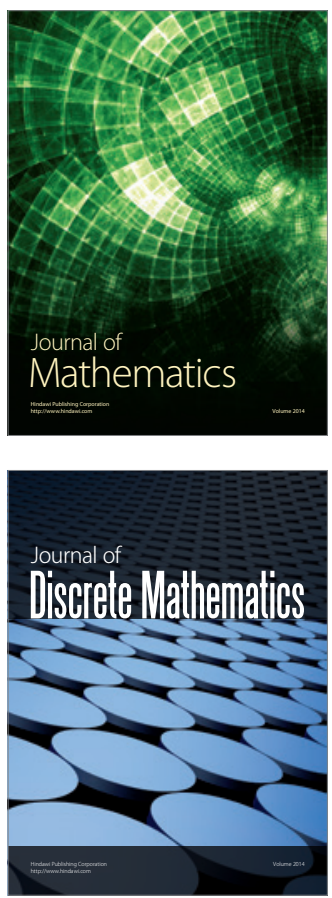

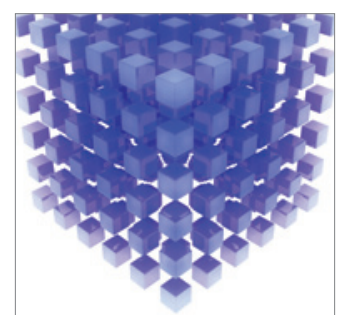

Mathematical Problems in Engineering
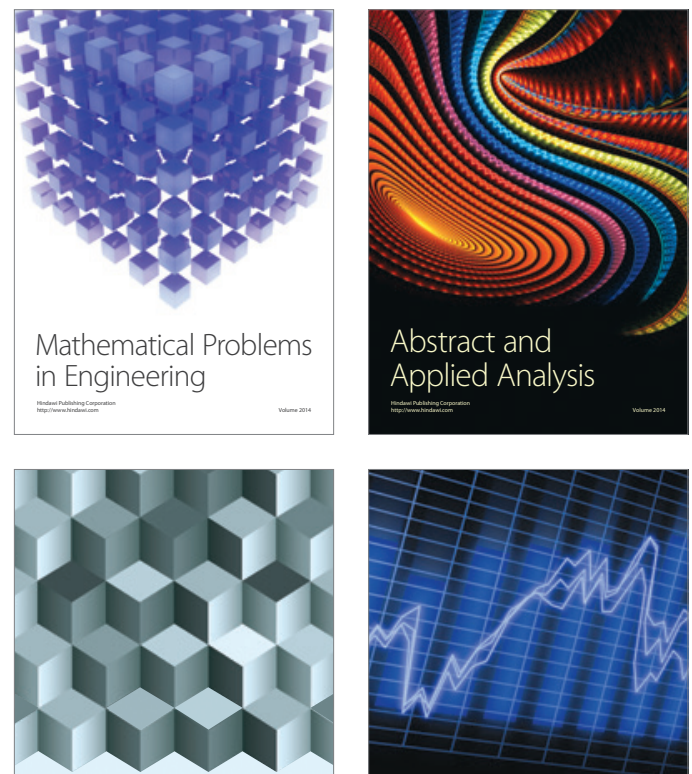

Journal of

Function Spaces

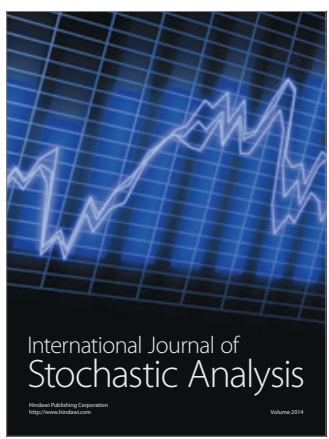

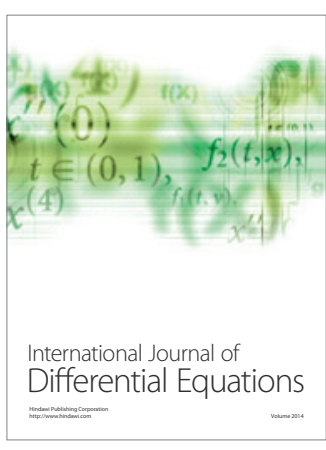
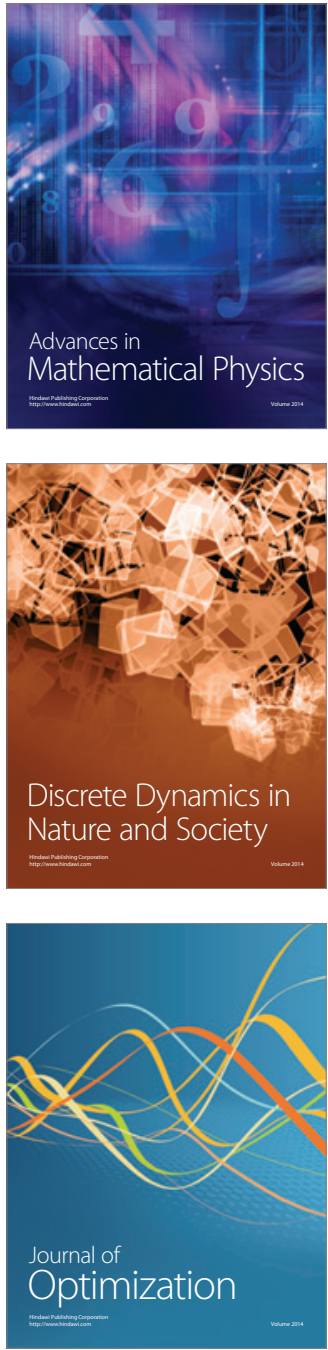